\title{
Endothelial growth medium suppresses apoptosis of mesenchymal stem cells in vitro via decrease of miR-29a
}

\author{
QIANQIAN WU $^{1}$, TAO FANG $^{2},{\text { MIN } \mathrm{CHEN}^{1} \text { and GUOXIAN QI }}^{1}$ \\ ${ }^{1}$ Department of Cardiology of Aging, Department of Cardiology, The First Affiliated Hospital of \\ China Medical University, Shenyang, Liaoning 110001; ${ }^{2}$ Department of Orthopedic Surgery, The \\ Shengjing Hospital of China Medical University, Shenyang, Liaoning 110004, P.R. China
}

Received July 21, 2016; Accepted May 5, 2017

DOI: $10.3892 / \mathrm{mmr} .2017 .6939$

\begin{abstract}
The administration of mesenchymal stem cells (MSCs) in cases of cardiac ischemia/reperfusion injury (IRI) has been associated with a significant reduction of myocardial cell death and an effective improvement in cardiac function. However, one major limiting factor in MSCs transplantation therapy is the low survival rate of the transplanted cells. The present study aimed to demonstrate that human amnion-derived mesenchymal stem cells (hAMSCs) cultured with endothelial growth medium (EGM-2) exhibited reduced apoptosis when exposed to serum-free and hypoxic conditions; and that the expression of microRNA (miR)-29a decreased significantly. Furthermore, miR-29a knockdown resulted in decreased apoptosis of hAMSCs and increased myeloid cell leukemia (MCL)-1 at the mRNA and protein levels. These results suggested that EGM-2 promoted survival of hAMSCs partly through the regulation of miR-29a and MCL-1 expression levels. These findings may provide a novel understanding of a potential effective therapeutic strategy for cardiac IRI.
\end{abstract}

\section{Introduction}

Mesenchymal stem cells (MSCs) represent a heterogeneous population of fibroblast-like multipotent cells which may differentiate into various mesodermal lineages and may be isolated from a number of tissues, including bone marrow, umbilical cord, amniotic membrane, or adipose tissue (1). Cardiac ischemia following acute myocardial infarction leads to impaired cardiac function and is associated with increased morbidity and mortality. MSCs administration in cases of cardiac ischemia/reperfusion injury (IRI) has been associated with a significant reduction of myocardial cell death and an

Correspondence to: Professor Guoxian Qi, Department of Cardiology of Aging, Department of Cardiology, The First Affiliated Hospital of China Medical University, 155 Nanjing North Street, Heping, Shenyang, Liaoning 110001, P.R. China

E-mail: qigx2002@medmail.com.cn

Key words: endothelial growth medium-2, mesenchymal stem cells, apoptosis, microRNA-29a, myeloid cell leukemia effective improvement in cardiac function $(2,3)$. However, a randomized control trial (4) and meta-analyses $(5,6)$ have demonstrated that improvement of cardiac function resulting from MSCs transplantation is limited. One major limiting factor in MSCs transplantation therapy is the low survival rate of the transplanted cells $(7,8)$. This is thought to be due to local inflammatory reactions, ischemia and hypoxia (9). Previous studies have focused on improving the survival of MSCs through various methods, including the use of genetically engineered MSCs, coupled with suitable tissue engineering materials, or preconditioning with optimal culture conditions (10-13).

microRNAs (miRs) are small (20-22 nucleotides long) noncoding RNAs that suppress protein translation by binding to target mRNAs, reducing their stability and/or inhibiting translation. Growing evidence indicates that miRs are involved in the regulation of cell survival, proliferation and migration, through mediating the expression of their target genes (14). Several miRs, including miR-29, miR-34a and miR-133, are involved in pathways modulating cellular apoptosis (15-17). However, the exact role of miRs in the survival of MSCs, in addition to the associated underlying mechanisms, remains to be elucidated.

Accordingly, the present study was designed to improve the survival of MSCs by cell culture. Specifically, it identified the expression of miR-29a in human amnion-derived (hA)MSCs cultured in different culture media. In addition, it also identified novel target genes of miR-29a and explored the underlying mechanisms associated with MSCs survival.

\section{Materials and methods}

Cell culture. Human amnion-derived (hA)MSCs were isolated from amniotic membranes of healthy donors using enzymatic digestion as previously described (18). All donors (between May 2015 and January 2016) gave their informed consent and the Ethics Committee of the First Affiliated Hospital of China Medical University (Shenyang, China) approved the study protocol. The hAMSCs were cultured in Dulbecco's modified Eagle's medium (DMEM; Hyclone; GE Healthcare Life Sciences, Logan, UT, USA) supplemented with $10 \%$ fetal bovine serum (FBS; Hyclone; GE Healthcare Life Sciences) and $100 \mathrm{U} / \mathrm{ml}$ penicillin and streptomycin (Hyclone; GE 
Healthcare Life Sciences) at $37^{\circ} \mathrm{C}$ in an incubator with $5 \% \mathrm{CO}_{2}$. For the endothelial cell culture conditions, cells were cultured in endothelial growth medium (EGM-2) with 5\% FBS and endothelial cell growth supplement (ECGS; Sciencell Research Laboratories, Inc., Carlsbad, CA, USA). All the subsequent assays were performed with 7-day cultured hAMSCs.

HAMSCs immunophenotyping. The immunophenotype of hAMSCs cultured in either hAMSCs culture conditions or EC culture conditions was analyzed using flow cytometry. A total of $1 \times 10^{6}$ cells were detached from culture dishes using trypsin solution (Hyclone; GE Healthcare Life Sciences) and stained with $5 \mu \mathrm{l}$ antibodies against cluster of differentiation (CD)31 (cat. no 303105), CD34 (cat. no 343505), CD73 (cat. no 344003), CD90 (cat. no 32810) and CD105 (cat. no 323205) (all undiluted; BioLegend, Inc., San Diego, CA, USA). Immunoglobulin $\mathrm{G}$ of the appropriate isotype was used as negative control. Data from 10,000 viable cells were acquired. List mode files were analyzed by FCS Express Software (version 3; BD Biosciences, Franklin Lakes, NJ, USA).

RNA isolation and reverse transcription-quantitative polymerase chain reaction ( $R T-q P C R)$. Total RNA was extracted from $1 \times 10^{6} \mathrm{hAMSCs}$ using TRIzol Reagent (Invitrogen; Thermo Fisher Scientific, Inc., Waltham, MA, USA) according to the manufacturer's protocol. RNA concentration was determined by a NanoDrop ND-1000 (Nano-Drop Technologies; Thermo Fisher Scientific, Inc., Wilmington, DE, USA). For miR-29a detection, poly A tail was added to RNase-free DNase digested total RNA using the poly A tailing kit (Ambion; Thermo Fisher Scientific, Inc.) according to the manufacturer's protocol. SYBR RT-qPCR was used to assay miRNA expression with the specific forward primers and the universal reverse primer complementary to the anchor primer. U6 primer was used as an miRNA internal control. For the mRNA analysis of myeloid cell leukemia (MCL)-1, cDNA was synthesized using PrimeScript $^{\mathrm{TM}}$ RT reagent (Takara Bio, Inc., Otsu, Japan) according to the manufacturer's protocol. Reactions were performed using the SYBR PrimeScript RT-PCR kit (Takara Bio, Inc.) with an ABI 7500 Sequence Detection System (Applied Biosystems; Thermo Fisher Scientific, Inc.) according to the manufacturer's protocol. The PCR reactions used for the amplification of miRNAs were conducted at $95^{\circ} \mathrm{C}$ for $30 \mathrm{sec}$, followed by 45 cycles of $95^{\circ} \mathrm{C}$ for $5 \mathrm{sec}$ and $60^{\circ} \mathrm{C}$ for $34 \mathrm{sec}$. As an internal control, $\beta$-actin level was quantified in parallel with the target genes. Normalization and fold-alterations were calculated using the $2^{-\Delta \Delta \mathrm{Cq}}$ method (19). All experiments were performed in triplicate and repeated three times. The primers used for RT-qPCR were as follows: Forward, 5'-TAGCACCAT CTGAAATCGGTTA-3' and reverse, 5'-GCTGTCAACGAT ACGCTACGT-3' for miR-29a; forward, 5'-CGCTTCGGC AGCACATATAC-3' and reverse, 5'-TTCACGAATTTGCGT GTCAT-3' for U6; forward, 5'-GAGGAGGACGAGTTGTAC CG-3' and reverse, 5'-CACAATCCTGCCCCAGTTTG-3' for MCL-1 and forward, 5'-AGGATTCCTATGTGGGCGAC-3' and reverse, 5'-ATAGCACAGCCTGGATAGCAA-3' for $\beta$-actin.

miR-29a overexpression and suppression. miR-29a mimic, negative control (nc), miR-29a inhibitor and inhibitor nc were purchased from Suzhou GenePharma Co. Ltd. (Suzhou, China) and the sequences were as follows: 5'-UAGCACCAU CUGAAAUCGGUUA-3' for miR-29a mimic; 5'-UAACCG AUUUCAGAUGGUGCUA-3' for miR-29a inhibitor; 5'-UUC UCCGAACGUGUCACGUTT-3' for NC; and 5'-CAGUAC UUUUGUGUAGUACAA-3' for inhibitor NC. Small interfering (si)RNA targeting human MCL-1 and control siRNA were obtained from Santa Cruz Biotechnology, Inc. (Dallas, TX, USA).

hAMSCs at $80 \%$ confluence were transfected with miR-29a mimic, inhibitor, MCL-1 siRNA and their corresponding controls (50 nM for each) using Lipofectamine 2000 (Invitrogen; Thermo Fisher Scientific, Inc.) according to the manufacturer's protocols. Following $48 \mathrm{~h}$, cells were incubated with serum-free DMEM with $300 \mu \mathrm{M}$ cobalt (II) chloride for a further $48 \mathrm{~h}$ and examined with Hoechst 33258 staining or flow cytometry.

Hoechst 33258 staining. Following cell incubation with serum-free DMEM with $300 \mu \mathrm{M}$ cobalt (II) chloride for 48 h, a Hoechst 33258 staining kit (Beyotime Institute of Biotechnology, Haimen, China) was used to observe the apoptotic cells induced by mimic or inhibitor of miR-29a, according to the manufacturer's protocol. Each assay was performed at least three times.

Caspases activities assay. Caspase 3 and 7 activities were determined using Apo-ONE ${ }^{\circledR}$ Homogeneous Caspase-3/7 Assay according to the manufacturer's protocols (cat. no G7792; Promega Corporation, Madison, WI, USA). The plate was read by a microplate reader at a wavelength of $405 \mathrm{~nm}$. Activities of caspases 3 and 7 were expressed as the ratio of treated cells to corresponding controls.

Flow cytometry. Following $48 \mathrm{~h}$ incubation in serum-free DMEM with $300 \mu \mathrm{M}$ cobalt (II) chloride, a FITC Annexin $\mathrm{V}$ Apoptosis Detection kit (BD Biosciences) was used to double stain cells with FITC-Annexin V and propidium iodide according to the manufacturer's protocols. A total of $2.5 \times 10^{5}$ cells were seeded into 6 -well plates for $48 \mathrm{~h}$ and removed using trypsin without EDTA, stained cells were analyzed using a flow cytometer (BD Biosciences). FCS Express Software (version 3; BD Biosciences) was used to observe cell apoptosis. In the graphs, cells were distinguished as dead, living, early apoptotic and late apoptotic cells. The aggregate of early and late apoptotic cells was regarded as an observation index to compare the experimental and negative groups. Each experiment was performed at least three times.

Luciferase reporter assay. The wild-type (WT) or mutant (MUT) 3'-untranslated region (UTR) segment of MCL-1 containing the putative miR-29a binding site was amplified and inserted into the pLUC Luciferase vector (Ambion; Thermo Fisher Scientific,Inc.). Site-directed mutagenesis of the miR-29a binding site in the MCL-1 3'-UTR was achieved with a commercially available kit (Beyotime Institute of Biotechnology). All the plasmids were confirmed by DNA sequencing. HEK293T cells (American Type Culture Collection, Manassas, VA, USA; $80 \%$ confluence) were co-transfected with $100 \mathrm{ng}$ reporter constructs and $50 \mathrm{nM}$ miR-29a mimic, inhibitor, or control 

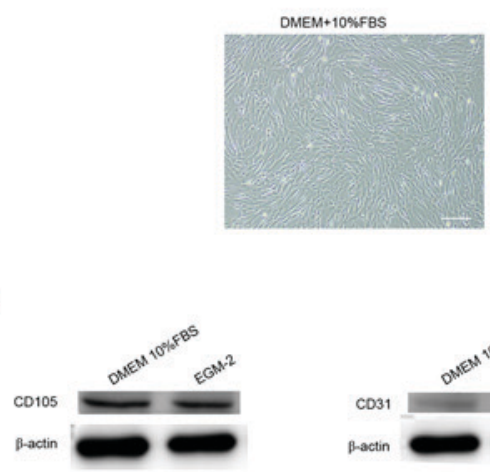

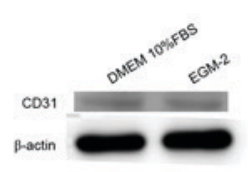

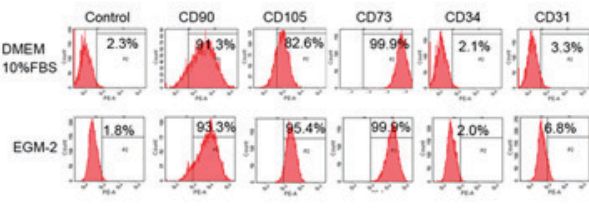

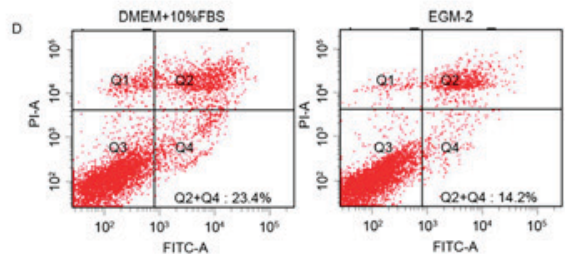

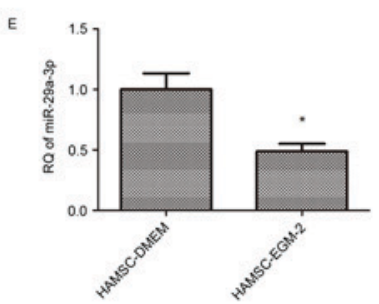

Figure 1. EGM-2 suppresses apoptosis of hAMSCs and downregulates miR-29a expression. (A) Representative photographs of hAMSCs cultured in DMEM and EGM-2; scale bars, $200 \mu \mathrm{m}$. (B) FACS analysis; surface marker of hAMSCs cultured in DMEM and EGM-2 compared with isotype-matched antibodies. (C) Western blot analysis of CD31 and CD105 expression in hAMSCs cultured in DMEM and EGM-2. (D) The rate of apoptotic hAMSCs cultured in DMEM or EGM-2. (E) Reverse transcription-quantitative polymerase chain reaction analysis of mature miR-29a expression in hAMSCs cultured in DMEM and EGM-2. EGM-2 decreased miR-29a and miR-29c expression. "P<0.05 vs. hAMSCs DMEM group. DMEM, Dulbecco's modified Eagle's medium; EGM-2, endothelial growth medium; hAMSCs, human amnion-derived mesenchymal stem cells; miR, microRNA; FACS, fluorescence-activated cell sorting; CD, cluster of differentiation; RQ, relative quality; FBS, fetal bovine serum.

miRNA using Lipofectamine 2000 (Invitrogen; Thermo Fisher Scientific, Inc.) according to the manufacturer's protocol. At $24 \mathrm{~h}$ posttransfection, cells were harvested and luciferase activity was assayed using the Dual Luciferase Reporter Assay System (Promega Corporation). A Renilla luciferase construct was used as the internal control. All experiments were repeated in triplicate.

Western blot analysis. Total proteins from hAMSC were harvested in lysis buffer (Beyotime Institute of Biotechnology) and quantified using the bicinchoninic acid method. Protein lysates were separated using 10\% SDS-acrylamide gels and transferred onto Protran nitrocellulose membranes (Whatman; GE Healthcare Life Sciences, Little Chalfont, UK). For immunodetection, membranes were incubated with antibodies directed against MCL-1 (1:1,000; cat. no ab32087; Abcam), CD31 (1:1,000; cat. no ab28364; Abcam), CD105 (1:1,000; cat. no ab107595; Abcam), or $\beta$-actin (1:10,000; cat. no ab8227; Abcam). Signals from horseradish-peroxidase-conjugated secondary antibodies (1:5,000; cat. no KC-RB-035; KangChen Bio-tech, Shanghai, China) were generated by enhanced chemiluminescence solution (ECL; GE Healthcare Life Sciences) and recorded on film. Quantification was performed using ImageJ software (version 1.45S; National institutes of Health, Bethesda, MD, USA). Experiments were repeated in triplicate.

Statistical analysis and bioinformatics. TargetScan (www.targetscan.org), PicTar (pictar.mdc-berlin.de) and miRanda (www.microrna.org) were used to predict the target genes of miR-29a. Statistical analysis was performed using SPSS software, version 13.0 (SPSS, Inc., Chicago, IL, USA). Data are expressed as the mean \pm standard deviation and were analyzed by a Student's t-test or one-way analysis of variance followed by Tukey's test. $\mathrm{P}<0.05$ was considered to indicate a statistically significant difference.

\section{Results}

Phenotype characterization of hAMSCs is variable depending on growth medium. hAMSCs cultured in DMEM supplemented with $10 \%$ FBS revealed a spindle fibroblast-like morphology, whereas those cultured in EGM-2 exhibited a cobblestone-like morphology (Fig. 1A). Flow cytometry revealed the expression of surface markers. hAMSCs cultured in the two types of medium were positive for CD73, CD90 and CD105, but were negative for CD31 and CD34 (Fig. 1B). Western blotting revealed that hAMSCs cultured in the two types of medium were positive for CD105 expression, and negative for CD31 expression (Fig. 1C).

EGM-2 culture decreases apoptosis of hAMSCs and miR-29a expression. Flow cytometry results demonstrated that the apoptosis of hAMSCs was significantly decreased in hAMSCs cultured in EGM-2 $(14.8 \pm 2.7 \%)$ compared with those cultured in DMEM (21.5 $\pm 3.1 \%$; Fig. 1D). Fig. 1E indicated that miR-29a expression was decreased in hAMSCs cultured in EGM-2 compared with hAMSCs cultured in DMEM ( 2-fold change).

Suppression of miR-29a decreases hAMSCs apoptosis. To evaluate the ability of miR-29a to control hAMSCs apoptotic activity, these cells were transfected by miR-29a mimic, inhibitor or their corresponding controls and incubated with serum-free DMEM with $300 \mu \mathrm{M}$ cobalt (II) chloride to undergo apoptosis. hAMSCs at $80 \%$ confluence were transfected with miR-29a inhibitor (50 and $100 \mathrm{nM}$ ) and $50 \mathrm{nM}$ inhibitor successfully suppressed the expression of miR-29a, so $50 \mathrm{nM}$ concentrations were selected for use in the present study (Fig. 2A). Following annexin-V/PI assays, miR-29a-transfected hAMSCs presented an apoptotic cell rate of $18.4 \pm 2.3 \%$ compared with hAMSCs transfected by the scramble oligos $(12.7 \pm 1.3 \%)$. Meanwhile, knocking-out miR-29a by transfection with inhibitor suppressed the 
A

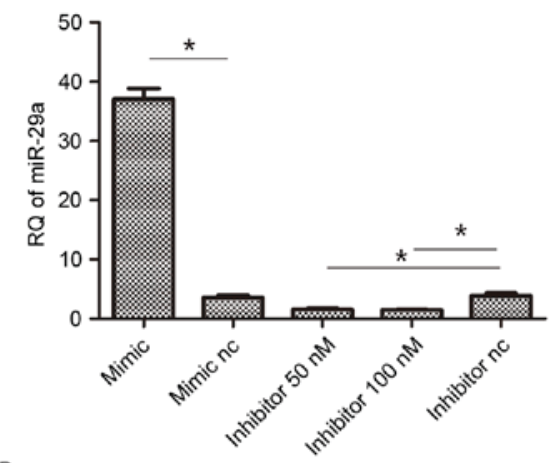

B
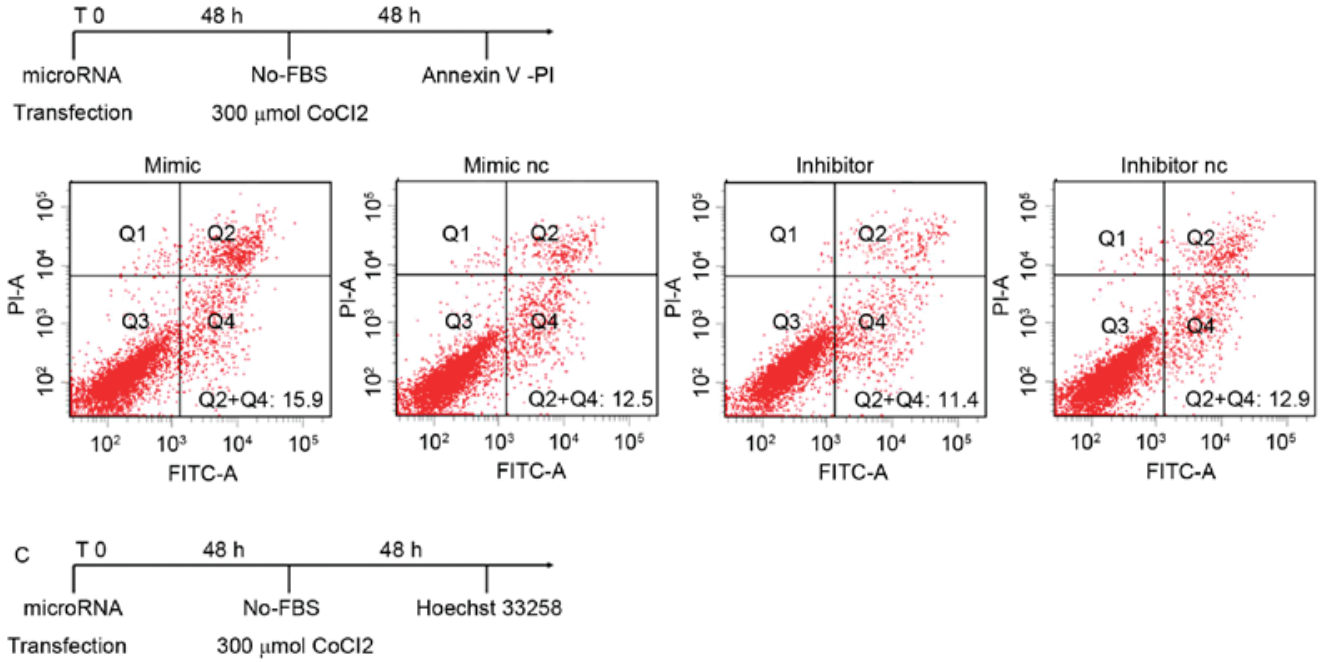

Transfection $\quad 300 \mu \mathrm{mol} \mathrm{CoCl} 2$
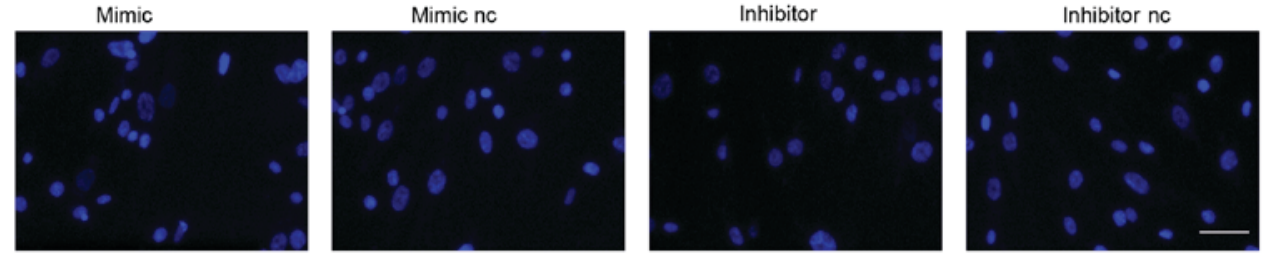

D

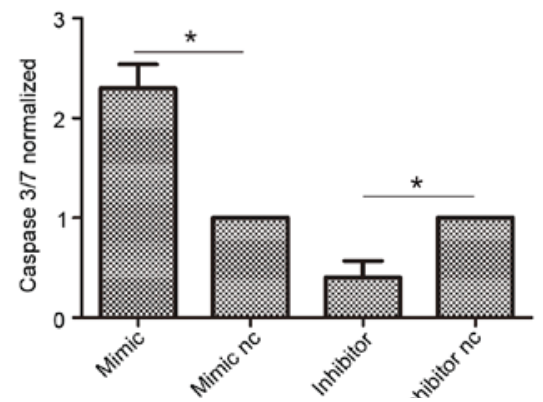

Figure 2. Influence of miR-29a on the apoptosis of hAMSCs. hAMSCs were transiently transfected with miR-29a-3p mimic, inhibitor and their corresponding controls for $48 \mathrm{~h}$. (A) Reverse transcription-quantitative polymerase chain reaction was performed to examine the relative miR-29a expression in hAMSCs. FBS-free medium with $\mathrm{CoCl} 2(300 \mu \mathrm{M})$ was then used for $48 \mathrm{~h}$ to induce apoptosis. (B) Annexin V/PI staining and (C) Hoechst staining were used to assess apoptosis; scale bar, $100 \mu \mathrm{m}$. (D) Caspase 3/7 activity assay to detect apoptotic level of mimic and inhibitor transfected cells. * $\mathrm{P}<0.05$. miR, microRNA; hAMSCs, human amnion-derived mesenchymal stem cells; RQ, relative quality; nc, negative control; CoCl2, cobalt (II) chloride.

apoptosis of hAMSCs $(9.1 \pm 1.8 \%$; Fig. 2B. The observations were confirmed by Hoechst 33258 staining, demonstrating a suppression of apoptosis in hAMSCs transfected with miR-29a inhibitor compared with the negative control (Fig. 2C).
These observations were confirmed by caspase 3/7 activities measurements, demonstrating an apoptosis suppression of hAMSCs transfected with inhibitor ( 2-fold) compared with the negative control (Fig. 2D). 


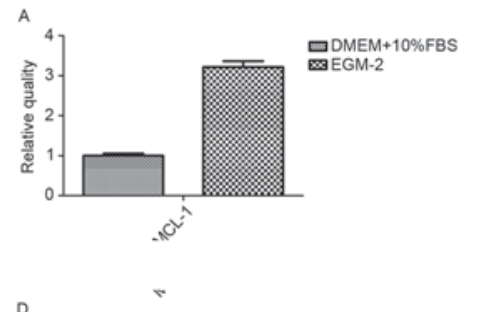

D

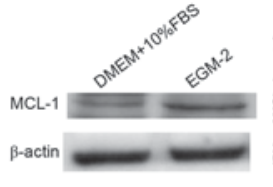

E
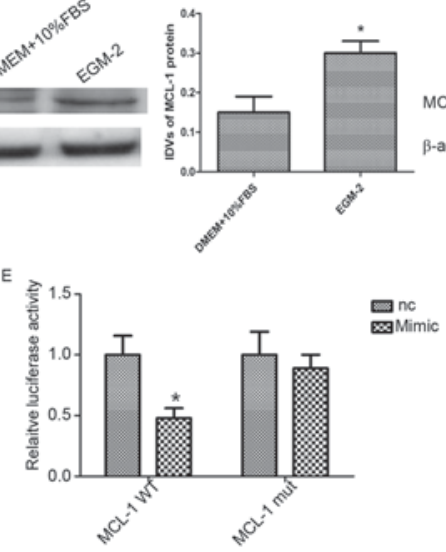

c
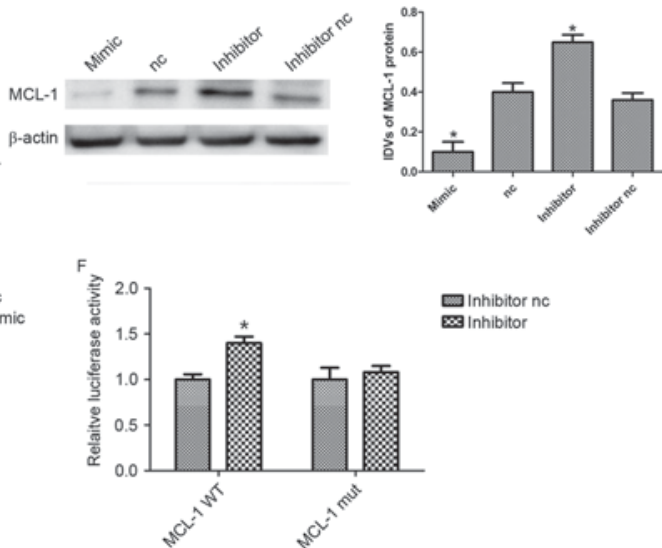

Figure 3. miR-29a targets MCL-1 in hAMSCs. (A) Reverse transcription-quantitative polymerase chain reaction analysis of MCL-1 expression in hAMSCs cultured in DMEM and EGM-2. (B) Western blot analysis of MCL-1 expression in hAMSCs cultured in DMEM and EGM-2. EGM-2 increased MCL-1 expression. $\beta$-actin was used as an endogenous control. Data are presented as the mean \pm standard deviation ( $\mathrm{n}=3 / \mathrm{group}$ ). (C) Western blot analysis of MCL-1 expression in miR-29a-3p mimic, inhibitor and their corresponding controls. $\beta$-actin was used as an endogenous control. Data are presented as the mean \pm standard deviation ( $\mathrm{n}=3$ /group). (D) Alignment of miR-29a with MCL-1 at the 3'-UTR and the mutation site of seed area. A luciferase reporter assay was performed to determine whether MCL-1 is a target of miR-29a. A WT or MUT of MCL-1 3'UTR was subcloned into the pLUC Luciferase vector. (E) Vector plus miR-29a mimics or (F) miR-29a inhibitor were co-transfected into hAMSCs. "P<0.05 vs. nc. miR, microRNA; MCL-1, myeloid cell leukemia-1; hAMSCs, human amnion-derived mesenchymal stem cells; EGM-2, endothelial growth medium; DMEM, Dulbecco's modified Eagle's medium; nc, negative control; WT, wildtype; MUT, mutated; IDV, integrated density value; FBS, fetal bovine serum.

MCL-1 is a target of miR-29a in hAMSCs. The expression of experimentally validated targets for miR-29a in hAMSCs were additionally examined. Potential targets of miR-29a were identified based on bioinformatics prediction. Through preliminary function screening, an anti-apoptotic protein, MCL-1, was selected to further study its interaction with miR-29a and its role in hAMSCs apoptosis. First, the basal level of MCL-1 mRNA in different culture mediums was observed by RT-qPCR. As presented in Fig. 3A, a significantly high level of MCL-1 mRNA was detected in the hAMSCs cultured in EGM-2 compared with DMEM $(\mathrm{P}<0.05)$. Similar results were obtained with western blotting analysis for MCL-1 protein expression (Fig. 3B). To ascertain if MCL-1 may be targeted by miR-29a, a gain-of-function experiment was performed with miR-29a in hAMSCs. MiR-29a or negative control was transiently transfected and endogenous MCL-1 expression was detected by western blotting. Densitometric measurement of the bands demonstrated that MCL-1 protein level was notably decreased in miR-29a overexpressing cells, whereas it appeared to be upregulated in miR-29a knockdown cells, compared with the control (Fig. 3C).

To confirm if miR-29a binds directly to the 3'-UTR of MCL-1 in HEK 293T cells, luciferase reporter vectors were constructed containing the WT or MUT 3'-UTR MCL-1 (Fig. 3D). miR-29a effectively reduced luciferase activity in 293 T cells transfected with the WT 3'-UTR of MCL-1. Conversely, miR-29a inhibitor significantly induced luciferase activity in cells transfected with WT MCL-1 3'UTR, whereas the MUT MCL-1 3'-UTR eliminated the suppression by miR-29a, compared with the control group (Fig. 3E and F). These findings indicated that MCL-1 is the direct target of miR-29a.

Furthermore, it was additionally determined whether the effect of miR-29a on hAMSCs apoptosis was via MCL-1. The mRNA levels of MCL-1 were knocked down (Fig. 4A and B). Following 48 h of co-transfection with siRNA and miR-29a inhibitor, apoptosis induction was conducted in each group. Then, FITC/PI assay, Caspase 3/7 activity assay and Hoechst 33258 staining assay were performed to determine cell apoptosis. As demonstrated in Fig. 4C, MCL-1 inhibition $(13.2 \pm 1.4 \%)$ reversed the function of miR-29a inhibitor $(9.7 \pm 1.6 \%)$ on cell apoptosis. The observations were confirmed by Hoechst 33258 staining (Fig. 4D) and Caspase 3/7 activity assay (Fig. 4E) demonstrating an apoptosis reduction with miR-29a inhibitor compared with controls. Taking these findings together, it is suggested that increased miR-29a results in increased levels of apoptosis via targeting of MCL-1 (decreasing its levels).

\section{Discussion}

The therapeutic potential of MSCs for cardiac repair is limited, partly due to their low survival rate within the ischemic myocardial microenvironment into which they are introduced $(8,9)$. Hypoxia and serum deprivation, imitating the ischemic engraftment environment, induce the apoptosis of MSCs (20). To improve cardiovascular cell therapy, continuous efforts have been made to improve their function. The present study demonstrated that hAMSCs cultured in EGM-2 conferred a protective effect against serum-free and hypoxic conditions. In the specific culture condition, hAMSCs expressed a lower level of miR-29a compared with the normal conditions. This downregulation contributed in part to the overexpression of MCL-1, the primary anti-apoptotic B-cell lymphoma 2 (Bcl-2) family member and thus, to the hAMSCs survival.

It was demonstrated that hAMSCs cultured in EGM-2 conferred a protective effect against serum-free and hypoxic conditions, making them suitable for cell therapy. In endothelial culture conditions, hAMSCs exhibited a cobblestone-like morphology, yet expressed similar levels of surface makers 

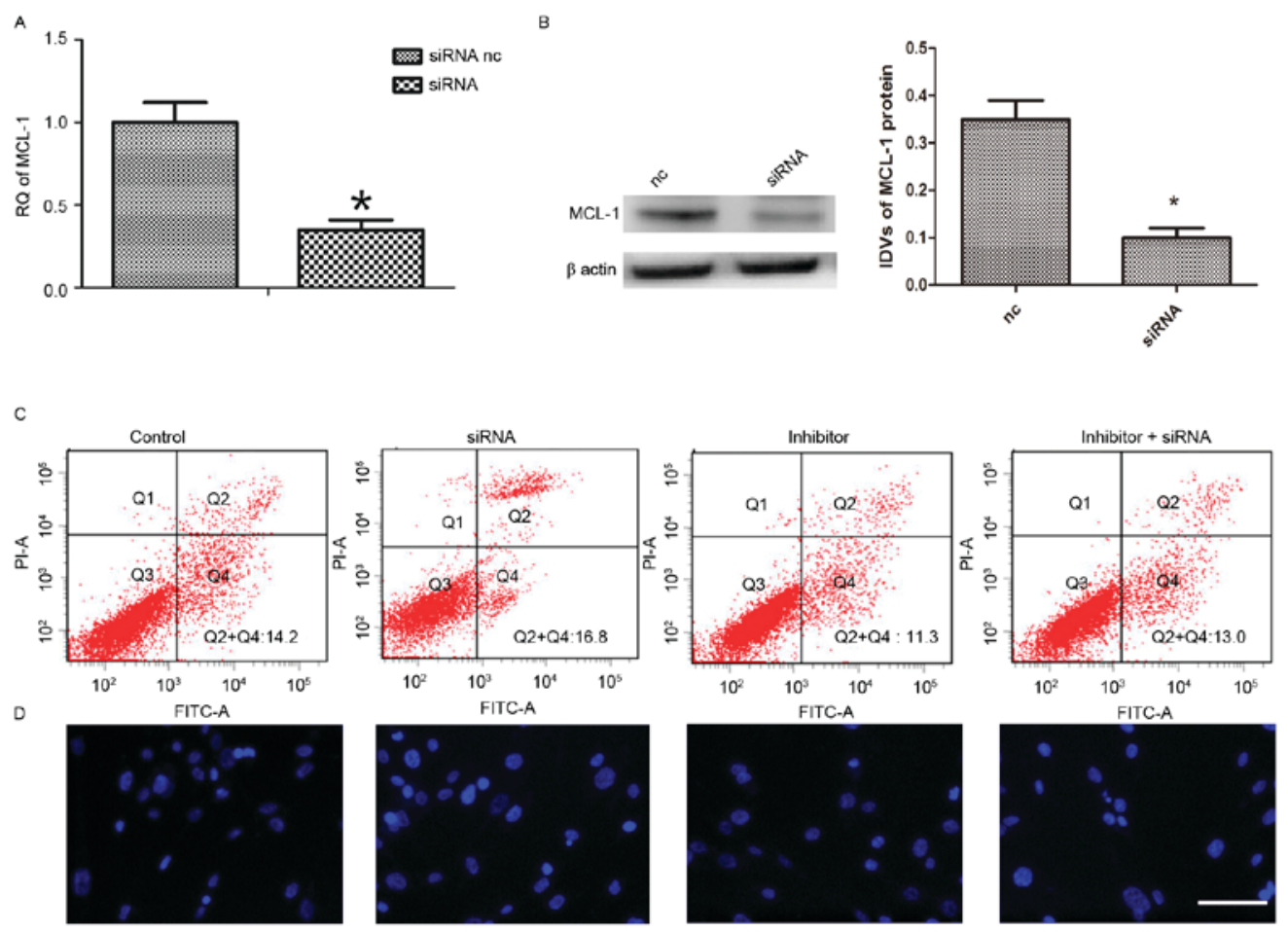

$\mathrm{E}$

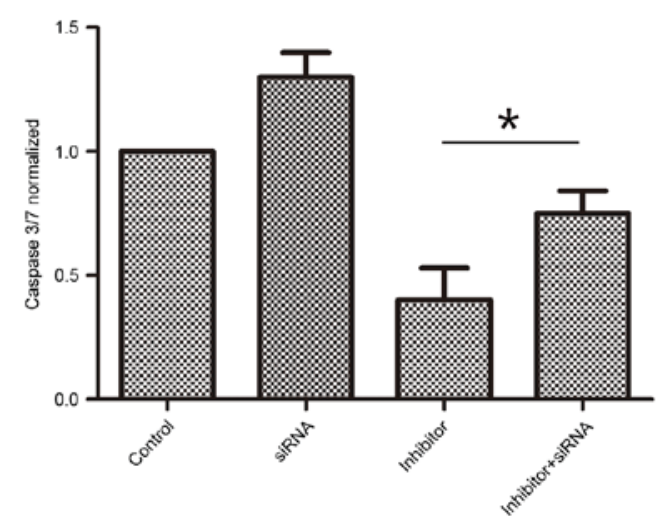

Figure 4. MCL-1 is required for the transcriptional activation of miR-29a by EGM-2. (A) Reverse transcription-polymerase chain reaction analysis and (B) western blotting analysis of MCL-1 expression in nc and MCL-1-siRNA hAMSCs. $\beta$-actin was used as an endogenous control. Data are presented as the mean \pm standard deviation ( $\mathrm{n}=3$ /group). (C) AnnexinV/PI assay, (D) Hoechst assay; scale bar, $100 \mu \mathrm{m}$ and (E) Caspase 3/7 activity assay. * $<0.05$ vs. negative control or as indicated. MCL-1, myeloid cell leukemia-1; miR, microRNA; EGM-2, endothelial growth medium; si, small interfering; nc, negative control; RQ, relative quality; IDV, integrated density value.

compared with hAMSCs cultured in DMEM. The present study is consistent with previous studies demonstrating that EGM-2 mediated the morphological alterations of hAMSCs. However, hAMSCs did not express the mature endothelial cell markers, von Willebrand factor and vascular endothelial-cadherin, and hAMSCs resisted undergoing a complete differentiation into mature endothelial cells $(21,22)$. Therefore, hAMSCs cultured in EGM-2 medium still possess stem cell characteristics and are unable to differentiate into endothelial cells in the EGM-2 medium. Using RT-qPCR, a significantly lower expression of miR-29a in EGM-2 was observed compared with cells cultured in DMEM. The present study is the first, to the authors' knowledge, to demonstrate the difference in expression of miR-29a in hAMSCs cultured with EGM-2. Therefore miR-29a was selected for the current study. Notably, miR-29a-inhibitor mediated protective effects on the apoptosis of hAMSCs induced by serum starvation and hypoxia. Results from the current study demonstrated that endogenous MCL-1 protein expression was markedly decreased in hAMSCs transfected with miR-29a.

It was demonstrated that MCL-1 is regulated at the posttranscriptional level by miR-29a in hAMSCs, thus suggesting a survival advantage in the silencing of miR-29a. MCL-1, in absence of $\mathrm{Bcl}-2$, promotes cell survival by inhibiting cell death $(23,24)$. It is known that miR-29 acts directly at the MCL-1 3'UTR. Mott et al (25) were the first to demonstrate in a cholangiocarcinoma cell model that MCL-1 may additionally be regulated at the posttranscriptional level by miR-29b. The results of the present study are in accordance with those reported by Mott et al (25), Garzon et al (26) and Xiong et al (27), they identified that miR-29a, another member of the miR-29 family, contributed to the downregulation of MCL-1 in hAMSCs. It was observed in the present study that 
targeted knockdown of MCL-1 by specific siRNA evidently inhibited the protective effect of miR-29a-inhibitor on hypoxiaand serum deprivation-induced apoptosis of hAMSCs.

However, there are studies suggesting that loss of miR-29 results in cell death. In diabetic nephropathy, increasing miR-29a action may protect against diabetic podocytopathy (28). In addition, in vivo evidence demonstrated the neuronal cell death of brain-specific knockdown of miR-29 (29). It therefore remains to be elucidated whether the apoptotic action of miR-29a is dependent on cell types or different culture conditions.

In conclusion, the present study demonstrated that hAMSCs experienced decreased apoptosis when cultured in EGM-2, partly through low expression of miR-29a, and this may provide a novel tool to improve stem cell therapy in the future.

\section{Acknowledgements}

The present study was supported by the Science and Technology Department of Liaoning Province (grant no 2013020200-206).

\section{References}

1. Parolini O, Alviano F, Bagnara GP, Bilic G, Bühring HJ, Evangelista M, Hennerbichler S, Liu B, Magatti M, Mao N, et al: Concise review: Isolation and characterization of cells from human term placenta: Outcome of the first international workshop on placenta derived stem cells. Stem cells 26: 300-311, 2008

2. Bollini S, Pozzobon M, Nobles M, Riegler J, Dong X, Piccoli M, Chiavegato A, Price AN, Ghionzoli M, Cheung KK, et al: In vitro and in vivo cardiomyogenic differentiation of amniotic fluid stem cells. Stem Cell Rev 7: 364-380, 2011.

3. Kim SW, Zhang HZ, Kim CE, Kim JM and Kim MH: Amniotic mesenchymal stem cells with robust chemotactic properties are effective in the treatment of a myocardial infarction model. Int J Cardiol 168: 1062-1069, 2013.

4. Katritsis DG, Sotiropoulou PA, Karvouni E, Karabinos I, Korovesis S, Perez SA, Voridis EM and Papamichail M: Transcoronary transplantation of autologous mesenchymal stem cells and endothelial progenitors into infarcted human myocardium. Catheter Cardiovasc Interv 65: 321-329, 2005.

5. Jeevanantham V, Butler M, Saad A, Abdel-Latif A, Zuba-Surma EK and Dawn B: Adult bone marrow cell therapy improves survival and induces long-term improvement in cardiac parameters: A systematic review and meta-analysis. Circulation 126: 551-568, 2012.

6. Hristov M, Heussen N, Schober A and Weber C: Intracoronary infusion of autologous bone marrow cells and left ventricular function after acute myocardial infarction: A meta-analysis. J Cell Mol Med 10: 727-733, 2006.

7. Toma C, Pittenger MF, Cahill KS, Byrne BJ and Kessler PD: Human mesenchymal stem cells differentiate to a cardiomyocyte phenotype in the adult murine heart. Circulation 105: 93-98, 2002.

8. Hua P, Liu JY, Tao J and Yang SR: Application and progress of combined mesenchymal stem cell transplantation in the treatment of ischemic cardiomyopathy. Biomed Res Int 2015: 568502, 2015.

9. Geng YJ: Molecular mechanisms for cardiovascular stem cell apoptosis and growth in the hearts with atherosclerotic coronary disease and ischemic heart failure. Ann N Y Acad Sci 1010 687-697, 2003.

10. Hamedi-Asl P, Halabian R, Bahmani P, Mohammadipour M, Mohammadzadeh M, Roushandeh AM, Jahanian-Najafabadi A, Kuwahara Y and Roudkenar MH: Adenovirus-mediated expression of the HO-1 protein within MSCs decreased cytotoxicity and inhibited apoptosis induced by oxidative stresses. Cell Stress Chaperones 17: 181-190, 2012.
11. Tang YL, Tang Y, Zhang YC, Qian K, Shen L and Phillips MI: Improved graft mesenchymal stem cell survival in ischemic heart with a hypoxia-regulated heme oxygenase-1 vector. J Am Coll Cardiol 46: 1339-1350, 2005.

12. He J, Wang C, Sun Y, Lu B, Cui J, Dong N, Zhang M, Liu Y and Yu B: Exendin-4 protects bone marrow-derived mesenchymal stem cells against oxygen/glucose and serum deprivation-induced apoptosis through the activation of the cAMP/PKA signaling pathway and the attenuation of ER stress. Int J Mol Med 37: 889-900, 2016.

13. Jin J, Jeong SI, Shin YM, Lim KS, Shin Hs, Lee YM, Koh HC and Kim KS: Transplantation of mesenchymal stem cells within a poly (lactide-co-epsilon-caprolactone) scaffold improves cardiac function in a rat myocardial infarction model. Eur J Heart Fail 11: 147-153, 2009.

14. Bartel DP: MicroRNAs: Genomics, biogenesis, mechanism and function. Cell 116: 281-297, 2004.

15. Ye Y, Hu Z, Lin Y, Zhang C and Perez-Polo JR: Downregulation of microRNA-29 by antisense inhibitors and a PPAR-gamma agonist protects against myocardial ischaemia-reperfusion injury. Cardiovasc Res 87: 535-544, 2010.

16. Zhang F, Cui J, Liu X, Lv B, Liu X, Xie Z and Yu B: Roles of microRNA-34a targeting SIRT1 in mesenchymal stem cells. Stem Cell Res Ther 6: 195, 2015.

17. Xu C, Lu Y, Pan Z, Chu W, Luo X, Lin H, Xiao J, Shan H, Wang $\mathrm{Z}$ and Yang B: The muscle-specific microRNAs miR-1 and miR-133 produce opposing effects on apoptosis by targeting HSP60, HSP70 and caspase-9 in cardiomyocytes. J Cell Sci 120: 3045-3052, 2007.

18. Liu X, Wang Z, Wang R, Zhao F, Shi P, Jiang Y and Pang X: Direct comparison of the potency of human mesenchymal stem cells derived from amnion tissue, bone marrow and adipose tissue at inducing dermal fibroblast responses to cutaneous wounds. Int J Mol Med 31: 407-415, 2013

19. Livak KJ and Schmittgen TD: Analysis of relative gene expression data using real-time quantitative PCR and the 2(-Delta Delta C(T)) method. Methods 25: 402-408, 2001.

20. Zhu W, Chen J, Cong X, Hu S and Chen X: Hypoxia and serum deprivation-induced apoptosis in mesenchymal stem cells. Stem cells 24: 416-425, 2006.

21. König J, Huppertz B, Desoye G, Parolini O, Fröhlich JD, Weiss G, Dohr G, Sedlmayr P and Lang I: Amnion-derived mesenchymal stromal cells show angiogenic properties but resist differentiation into mature endothelial cells. Stem Cells Dev 21: 1309-1320, 2012.

22. König J, Weiss G, Rossi D, Wankhammer K, Reinisch A, Kinzer M, Huppertz B, Pfeiffer D, Parolini O and Lang I: Placental mesenchymal stromal cells derived from blood vessels or avascular tissues: What is the better choice to support endothelial cell function? Stem Cells Dev 24: 115-131, 2015.

23. Opferman JT, Letai A, Beard C, Sorcinelli MD, Ong CC and Korsmeyer SJ: Development and maintenance of B and T lymphocytes requires antiapoptotic MCL-1. Nature 426: 671-676, 2003.

24. Maurer U, Charvet C, Wagman AS, Dejardin E and Green DR: Glycogen synthase kinase-3 regulates mitochondrial outer membrane permeabilization and apoptosis by destabilization of MCL-1. Mol Cell 21: 749-760, 2006.

25. Mott JL, Kobayashi S, Bronk SF and Gores GJ: Mir-29 regulates Mcl-1 protein expression and apoptosis. Oncogene 26: 6133-6140, 2007.

26. Garzon R, Heaphy CE, Havelange V, Fabbri M, Volinia S, Tsao T, Zanesi N, Kornblau SM, Marcucci G, Calin GA, et al: MicroRNA 29b functions in acute myeloid leukemia. Blood 114: 5331-5341, 2009

27. Xiong Y, Fang JH, Yun JP, Yang J, Zhang Y, Jia WH and Zhuang SM: Effects of microRNA-29 on apoptosis, tumorigenicity and prognosis of hepatocellular carcinoma. Hepatology 51: 836-845, 2010.

28. Lin CL, Lee PH, Hsu YC, Lei CC, Ko JY, Chuang PC, Huang YT, Wang SY, Wu SL, Chen YS, et al: MicroRNA-29a promotion of nephrin acetylation ameliorates hyperglycemia-induced podocyte dysfunction. J Am Soc Nephrol 25: 1698-1709, 2014.

29. Roshan R, Shridhar S, Sarangdhar MA, Banik A, Chawla M, Garg M, Singh VP and Pillai B: Brain-specific knockdown of miR-29 results in neuronal cell death and ataxia in mice. RNA 20: 1287-1297, 2014. 